\section{Efficacy and Safety of Direct Oral Anticoagulants in Venous Thromboembolism Compared to Traditional Anticoagulants in Morbidly Obese Patients: A Systematic Review and Meta-Analysis}

\author{
Anjan Katel ${ }^{1}$, Madan Aryal ${ }^{2}$, Arun Neupane ${ }^{3}$, Rohit Gosain ${ }^{4}$, Ranjan Pathak ${ }^{5}$, Yashoda Bhandari ${ }^{6}$, \\ Peter Kouides ${ }^{7}$ \\ 1. Department of Internal Medicine, Kathmandu University School of Medical Sciences, Dhulikhel, NPL 2. Department \\ of Medicine, Enloe Medical Center, Enloe Regional Cancer Center, California, USA 3. Department of Nursing and \\ Critical Care, Alta Bates Summit Medical Center, California, USA 4. Department of Medicine, Roswell Park \\ Comprehensive Cancer Institute, New York, USA 5. Department of Oncology, Nepal Medical College Pvt. Ltd., \\ Kathmandu, NPL 6. Department of Nursing, University at Buffalo, New York, USA 7. Department of \\ Hematology/Oncology, Rochester General Hospital, Rochester, USA
}

Corresponding author: Anjan Katel, anjan.katel3@gmail.com

\section{Abstract \\ Background}

Randomized clinical trials comparing the efficacy and safety of direct oral anticoagulants (DOAC) with vitamin K antagonist (VKA) or low molecular weight heparin (LMWH) for the treatment of venous thromboembolism (VTE) generally exclude patients who are morbidly obese (body mass index $\geqslant 40 \mathrm{~kg} / \mathrm{m}^{2}$ or weight $\geqslant 120 \mathrm{~kg}$ ). Recently, smaller studies have compared DOACs with warfarin or low molecular weight heparin (LMWH) in morbidly obese patients with VTE. We aim to systematically review and do a metaanalysis of the studies that directly compared DOACs with VKAs or LMWH in morbidly obese patients.

\section{Methods}

Studies comparing DOAC with warfarin or LMWH in patients with acute VTE were identified through electronic literature searches of MEDLINE, EMBASE, Scopus, clinicaltrials.gov, and the Cochrane Library up to March 2020. The primary efficacy outcome was recurrent VTE and the primary safety outcome was major bleeding as defined by the International Society on Thrombosis and Haemostasis (ISTH) guidelines. Studyspecific odds ratios (OR) were calculated and combined using a random-effects model meta-analysis.

\section{Result}

Five studies were identified. Recurrent VTE occurred in 95 of 3207 (2.96\%) patients in the DOAC group and 81 of 3181 (2.54\%) patients in the VKA and LMWH group (OR: 1.17 ; $95 \%$ CI 0.87 to $1.59, \mathrm{p}=.30$ ). Major bleeding occurred in 63 of 3316 (1.89\%) patients in the DOAC group, and 83 of 3259(2.54\%) patients in the VKA or LMWH group (OR: 0.74; 95\% CI: 0.53 to 1.03, p=.08). Sensitivity analysis comparing factor Xa inhibitors apixaban and rivaroxaban to warfarin also yielded consistent findings.

\section{() Copyright 2021}

Katel et al. This is an open access article distributed under the terms of the Creative Commons Attribution License CC-BY 4.0., which permits unrestricted use, distribution, and reproduction in any medium, provided the original author and source are credited.

\section{Conclusion}

DOACs showed similar efficacy and safety in the prevention of recurrent VTE risk and major bleeding events in morbidly obese patients when compared to warfarin/LMWH. Our study underscores the need for further modifications of therapy to reduce the high VTE recurrence rate irrespective of whether the patient is on a DOAC or VKA. This might be possible through a very large multi-institutional randomized clinical trial.

Categories: Endocrinology/Diabetes/Metabolism, Internal Medicine, Hematology

Keywords: morbid obesity, doacs, vte prophylaxis, vitamin k antagonists, non-vitamin k oral anticoagulant

\section{Introduction}

Direct oral anticoagulants (DOACs), such as apixaban and rivaroxaban, are becoming preferred agents in the treatment of acute venous thromboembolism (VTE) as compared with vitamin K antagonists (VKA) due to numerous advantages, including fewer monitoring requirements, fewer dietary restrictions, and fixed dosing [1-2]. Data from clinical trials in patients with acute VTE and non-valvular atrial fibrillation (NVAF) have shown that DOACs have similar efficacy in the treatment of VTE as compared with VKAs with a similar or lower bleeding risk [3].

Obesity is a pro-inflammatory and prothrombotic state [4] and an independent risk factor for VTE, increasing the risk of VTE by two to six-fold [5]. Due to alterations in pharmacokinetic parameters including 
volume of distribution, half-life, and clearance, anticoagulant medication doses often require adjustments in obese patients [6-7]. Although there are limited data evaluating the efficacy of DOACs in non-morbidly obese patients (body mass index (BMI) $<40 \mathrm{~kg} / \mathrm{m} 2$ ) in the treatment of VTE, less information exists to guide the treatment of morbidly obese patients (BMI $\geqslant 40 \mathrm{~kg} / \mathrm{m}^{2}$ or weight $\geqslant 120 \mathrm{~kg}$ ) [8] since they were underrepresented in clinical trials. Furthermore, the adequacy of fixed-dose anticoagulation in this population has been questioned due to concerns about efficacy [9]. The International Society on Thrombosis and Haemostasis (ISTH) recommends avoiding DOACs in morbidly obese patients and closely monitoring plasma drug levels if used [7]. Similarly, laboratory monitoring of anti-Xa while using LMWH in morbidly obese populations has been suggested [10]. However, plasma drug monitoring for DOACs may not be readily available, thus limiting the use of DOACs in these patients despite patient preferences.

We, therefore, sought to perform a systematic review and meta-analysis to examine the efficacy and safety of DOACs in morbidly obese patients in the treatment of VTE in the reported literature to date.

\section{Materials And Methods}

The Preferred Reporting Items for Systematic Reviews and Meta-Analyses (PRISMA) statement for reporting systematic reviews as recommended by the Cochrane Collaboration was followed in this systematic review [11].

\section{Literature search}

A systematic literature review using MEDLINE, EMBASE, Cochrane CENTRAL, Scopus, and clinicaltrials.gov as performed from each database inception to March 2020.

The search was restricted to English-language publications and was performed applying all potential synonyms of four broad themes: "direct oral anticoagulants," "warfarin," "factor Xa inhibitors," and "low molecular weight heparins," and combined using the Boolean operator "AND.” References from review articles, editorials, and conference publications were hand-searched and cross-referenced to ensure a comprehensive search.

\section{Study selection}

We included all studies that evaluated the treatment of acute VTE in adult (age $\geqslant 18$ ) obese patients that compared rivaroxaban, apixaban, edoxaban, or dabigatran with VKA or LMWH and reported the incidence of VTE or acute bleeding in the first 12 months.

Studies of DOACs in NVAF were not included. In addition, studies in VTE that did not report VTE and major bleeding outcomes separately were also excluded [12]. The study by Coons et al. was not included due to a lack of separate data on morbidly obese patients [9]. Studies that evaluated both VTE and NVAF patients, but did not report data separately were also excluded [13-14]. Two studies were not included, as both intervention and control arms were DOACs $[12,15]$. Prior studies comparing DOACs in obesity have been listed in Table 1.

\begin{tabular}{|c|c|c|c|c|c|}
\hline & $\begin{array}{l}\text { Kushnir et } \\
\text { al, } 2019\end{array}$ & $\begin{array}{l}\text { Perales et } \\
\text { al, } 2019\end{array}$ & $\begin{array}{l}\text { Spyropoulos et } \\
\text { al, } 2019\end{array}$ & Sa et al, 2019 & Quan et al, 2020 \\
\hline Source type & Journal article & $\begin{array}{l}\text { Journal } \\
\text { article }\end{array}$ & Journal article & Journal article & Journal article \\
\hline Study design & $\begin{array}{l}\text { Single-center, } \\
\text { retrospective } \\
\text { analysis of chart } \\
\text { data Montefiore } \\
\text { Medical Center } \\
\text { (Bronx, NY, USA) }\end{array}$ & $\begin{array}{l}\text { Retrospective } \\
\text { chart } \\
\text { review at } 2 \\
\text { academic } \\
\text { medical } \\
\text { centers }\end{array}$ & $\begin{array}{l}\text { Retrospective } \\
\text { 1:1, propensity } \\
\text { score-matched } \\
\text { cohort study } \\
\text { using } 2 \text { US } \\
\text { claim database }\end{array}$ & $\begin{array}{l}\text { Retrospective, } \\
\text { single-center } \\
\text { cohort study in } \\
\text { London (Canada) }\end{array}$ & $\begin{array}{l}\text { Sub-study of retrospective chart review } \\
\text { at Canadian institutions }\end{array}$ \\
\hline $\begin{array}{l}\text { Comparison } \\
\text { made }\end{array}$ & A Vs R VS W & R Vs W & R vs W & DOAC vs W & DOAC vs TT \\
\hline $\begin{array}{l}\text { Inclusion } \\
\text { criteria }\end{array}$ & $\begin{array}{l}\text { BMI } \geq 40, \geq 18 \\
\text { years old, } \\
\text { Included AF and } \\
\text { VTE (Only VTE } \\
\text { data taken) }\end{array}$ & $\begin{array}{l}\text { BMl>40, } \\
>120 \mathrm{~kg} \\
\text { Included AF } \\
\text { and VTE (only } \\
\text { VTE data } \\
\text { taken) }\end{array}$ & $\begin{array}{l}\text { ICD 9/ICD } 10 \\
\text { diagnosis code } \\
\text { for morbid } \\
\text { obesity. } \\
\text { Included VTE } \\
\text { patients }\end{array}$ & $\begin{array}{l}\mathrm{BMI} \geq 30 \mathrm{~kg} / \mathrm{m}^{2} \\
\text { versus }<30 \mathrm{~kg} / \mathrm{m}^{2} \\
\text { Weight } \geq 120 \mathrm{~kg} \text { vs. } \\
<120 \mathrm{~kg}(\text { Only data } \\
\text { from weight } \geq 120 \\
\mathrm{~kg} \text { included })\end{array}$ & Weight >120kg, VTE only \\
\hline & $53 \mathrm{~A}$ & $56+/-14 \mathrm{R}$ & $53 \mathrm{R}$ & NS & 53 DOAC \\
\hline
\end{tabular}




\section{Cureus}

\begin{tabular}{|c|c|c|c|c|c|}
\hline \multirow{2}{*}{$\begin{array}{l}\text { Mean age, } \\
\text { years }\end{array}$} & $52 \mathrm{R}$ & $55+/-15 \mathrm{~W}$ & \multicolumn{2}{|l|}{$53 \mathrm{~W}$} & \multirow[t]{2}{*}{$52 \mathrm{TT}$} \\
\hline & $58 \mathrm{~W}$ & NS & NS & & \\
\hline \multirow{3}{*}{ Female sex, \% } & $74.4 \mathrm{~A}$ & $48 \mathrm{R}$ & $60.5 \mathrm{R}$ & NS & 34 DOAC \\
\hline & $65.7 \mathrm{R}$ & $44.5 \mathrm{~W}$ & $60.2 \mathrm{~W}$ & & $35.9 \mathrm{TT}$ \\
\hline & $70.6 \mathrm{~W}$ & & & & \\
\hline \multirow{3}{*}{$\begin{array}{l}\text { Mean BMI } \\
\left(\mathrm{kg} / \mathrm{m}^{2}\right)\end{array}$} & $43.3 \mathrm{~A}$ & $45 \mathrm{R}$ & NS & NS & NS \\
\hline & $43.7 \mathrm{R}$ & $44 \mathrm{~W}$ & & & \\
\hline & $45.3 \mathrm{~W}$ & & & & \\
\hline \multirow{2}{*}{$\begin{array}{l}\text { Mean weight, } \\
\text { kg }\end{array}$} & \multirow{2}{*}{ NS } & \multirow{2}{*}{$133 \mathrm{R}, 134 \mathrm{~W}$} & \multirow[t]{2}{*}{ NS } & \multirow[t]{2}{*}{ NS } & $138 \mathrm{DOAC}$ \\
\hline & & & & & $142 \mathrm{TT}$ \\
\hline Medications, \% & NS & & NS & NS & NS \\
\hline $\begin{array}{l}\text { Antiplatelet } \\
\text { agents }\end{array}$ & & $\begin{array}{l}\text { ASA: 34/84 R, } \\
\text { 35/92 W } \\
\text { Clopidogrel: } \\
\text { 4/84 R, 8/92 } \\
\text { W NSAID: } \\
\text { 9/84 R, 3/92 } \\
\text { W }\end{array}$ & & & \\
\hline Comorbidities & $\begin{array}{l}\text { Charlson } \\
\text { Comorbidity } \\
\text { Index 0 A } 1 \text { R } 2 \\
\text { W }\end{array}$ & $3 \mathrm{R}, 3 \mathrm{~W}$ & $1.2 \mathrm{R}, 1.2 \mathrm{~W}$ & NS & \\
\hline Malignancy & NS & $\begin{array}{l}3 / 84 \mathrm{R}, 7 / 92 \\
W\end{array}$ & $\begin{array}{l}\text { 211/2890, } \\
199 / 2890\end{array}$ & & 3/109 DOAC, 16/78 TT \\
\hline Chronic kidney & NS & $\begin{array}{l}0 / 84 \mathrm{~A}, 10 / 92 \\
W\end{array}$ & $\begin{array}{l}182 / 2890 \\
(6.3 \%), R, \\
203 / 2890 \\
(7.0 \%) W\end{array}$ & & \\
\hline $\begin{array}{l}\text { Total duration } \\
\text { of study, } \\
\text { mo Follow-up }\end{array}$ & $\begin{array}{l}163 \text { days }(\mathrm{A}), 217 \\
\text { days }(\mathrm{R}), 206 \\
\text { days }(\mathrm{W})\end{array}$ & 12 months & $\begin{array}{l}10 \text { months } R, \\
10.5 \text { months } W\end{array}$ & 1 year & 1 year \\
\hline \multirow{4}{*}{$\begin{array}{l}\text { Study groups } \\
\text { (total no. of } \\
\text { patients in each } \\
\text { group) }\end{array}$} & 3 groups & 2 groups & 2 groups & 2 groups & 2 groups \\
\hline & $47 \mathrm{~A}$ & $84 \mathrm{R}$ & $2890 \mathrm{R}$ & 71 DOAC & DOAC 109 \\
\hline & $152 \mathrm{R}$ & $92 \mathrm{~W}$ & $2890 \mathrm{~W}$ & $62 \mathrm{~W}$ & TT 78 \\
\hline & $167 \mathrm{~W}$ & & & & \\
\hline $\begin{array}{l}\text { Primary } \\
\text { outcome }\end{array}$ & $\begin{array}{l}\text { Recurrent VTE, } \\
\text { Major bleeding }\end{array}$ & $\begin{array}{l}\text { VTE } \\
\text { recurrence } \\
\text { Mortality }\end{array}$ & $\begin{array}{l}\text { VTE recurrence } \\
\text { Major bleeding }\end{array}$ & VTE recurrence & Overall rate of recurrent VTE \\
\hline $\begin{array}{l}\text { Secondary } \\
\text { outcome }\end{array}$ & $\begin{array}{l}\text { Composite } \\
\text { bleeding }\end{array}$ & $\begin{array}{l}\text { Length of stay } \\
\text { and bleeding } \\
\text { complications }\end{array}$ & $\begin{array}{l}\text { Major Bleeding } \\
\text { risk Healthcare } \\
\text { resource } \\
\text { utilization and } \\
\text { costs }\end{array}$ & Major bleeding & $\begin{array}{l}\text { Rate of recurrent VTE on and off } \\
\text { anticoagulation therapy, dosing } \\
\text { regimens used, duration of therapy, } \\
\text { bleeding events, and proportions of } \\
\text { patients prescribed a DOAC only vs TT } \\
\text { only vs having therapy switches. }\end{array}$ \\
\hline \multirow{3}{*}{$\begin{array}{l}\text { Recurrent VTE, } \\
\%\end{array}$} & $2.1 \mathrm{~A}$ & & $\begin{array}{l}\text { Risk- R } 16.8 \% \\
\text { (485/2890) }\end{array}$ & 2.81 DOAC & \multirow{3}{*}{0.006 events per patient year } \\
\hline & $2.0 \mathrm{R}$ & $4.2 \mathrm{R}$ & $\begin{array}{l}\text { Risk- W 15.9\% } \\
\text { (459/2890) }\end{array}$ & $0 \mathrm{~W}$ & \\
\hline & & & Actual- R & & \\
\hline
\end{tabular}




\section{Cureus}

\begin{tabular}{|c|c|c|c|c|c|}
\hline & $1.2 \mathrm{~W}$ & $6.4 \mathrm{~W}$ & $\begin{array}{l}\text { PPPY Actual- } \\
\text { W-0.03 PPPY }\end{array}$ & & \\
\hline \multirow[t]{2}{*}{$\begin{array}{l}\text { Major bleeding, } \\
\%\end{array}$} & $\begin{array}{l}2.1 \mathrm{~A} \\
1.3 \mathrm{R}\end{array}$ & $6.3 \mathrm{R}$ & $1.8 \mathrm{R}$ & 1.4 DOAC & 3.6 DOAC \\
\hline & $2.4 \mathrm{~W}$ & $3.2 \mathrm{~W}$ & $2.5 \mathrm{~W}$ & $1.6 \mathrm{~W}$ & $3.8 \mathrm{TT}$ \\
\hline $\begin{array}{l}\text { Nonmajor/minor } \\
\text { bleeding, \% }\end{array}$ & $\begin{array}{l}\text { NS (composite } \\
\text { bleeding) } 10.7 \mathrm{~A} \\
9.8 \mathrm{R} 16.4 \mathrm{~W}\end{array}$ & NS & NS & NS & NS \\
\hline $\begin{array}{l}\text { Study outcomes } \\
\text { reported at }\end{array}$ & Within 6 months & $\begin{array}{l}\text { Within } 12 \\
\text { months }\end{array}$ & $\begin{array}{l}\text { Time to first } \\
\text { major bleeding } \\
\text { event- R } 69 \\
\text { days (mean } 83 \text { ), } \\
\text { W-77 days } \\
\text { (mean } 79 \text { ) }\end{array}$ & Within 1 year & Within 12 months \\
\hline
\end{tabular}

\section{TABLE 1: Baseline characteristics of included studies}

A, apixaban; ARR, absolute risk reduction; CRNMB, clinically relevant non-major bleeding; NS, not specified; R, rivaroxaban; VA, Veterans Affairs; W, warfarin; PPPY, per patient per year, DOAC: directly acting anticoagulants (including apixaban, rivaroxaban, and dabigatran), TT: traditional therapy (including warfarin and low molecular weight heparin)

In case of multiple publications resulting from the same study, we included data from the most recent publication. Two investigators (R.G. and A.K.) screened and retrieved relevant articles and excluded irrelevant studies. Relevant data were extracted by two investigators (R.G. and A.K) and checked by two other authors (A.N. and Y.B.). Two additional investigators (M.R.A. and R.P.) participated in the review process when uncertainty about eligibility criteria arose.

\section{Outcomes and data extraction}

The primary efficacy outcome of interest was the rate of recurrent VTE (composite of any recurrent deep vein thrombosis or pulmonary embolism) in the first 12 months; the primary safety outcome was major bleeding.

Recurrent VTE was defined as positive imaging findings on ultrasound Doppler or computed tomography. Major bleeding was defined as bleeding events requiring intervention or transfusion, cardiac tamponade, or pericardial effusion requiring drainage, retroperitoneal bleeding, intracranial bleed, massive hemoptysis, hemithorax, bleeding requiring an extra hospital stay, and patients requiring hospitalization as per the ISTH guidelines [16]. Rates of minor bleeding were not included due to significant missing data.

The following details from each study were extracted and tabulated: study design, modes of comparison, mean age, percentage of female population, mean BMI, mean weight, medications, comorbidities number of patients with acute VTE, estimated recurrent VTE risk, estimated major bleeding risk, duration of anticoagulation, and follow-up period.

\section{Risk of bias}

We assessed the risk-of-bias using a modified Newcastle-Ottawa Quality Assessment Scale for retrospective studies [17].

\section{Statistical analysis}

Outcomes from the individual studies were aggregated with RevMan (version 5.3, Cochrane Collaboration, Oxford, United Kingdom) applying the Mantel-Haenszel test. Odds ratios (ORs) and 95\% confidence intervals (CIs) were estimated using a random-effects method to account for the presence of variability among the studies. The $\mathrm{I}^{2}$ statistic was used to assess heterogeneity. Two-tailed $\mathrm{p}$-values $<.05$ were considered to indicate statistical significance.

\section{Results}

\section{Study selection}

The literature search identified 509 unique references. After a full-text review of 26 articles, we identified five observational studies for qualitative and quantitative analysis (Figure 1). 


\section{Cureus}

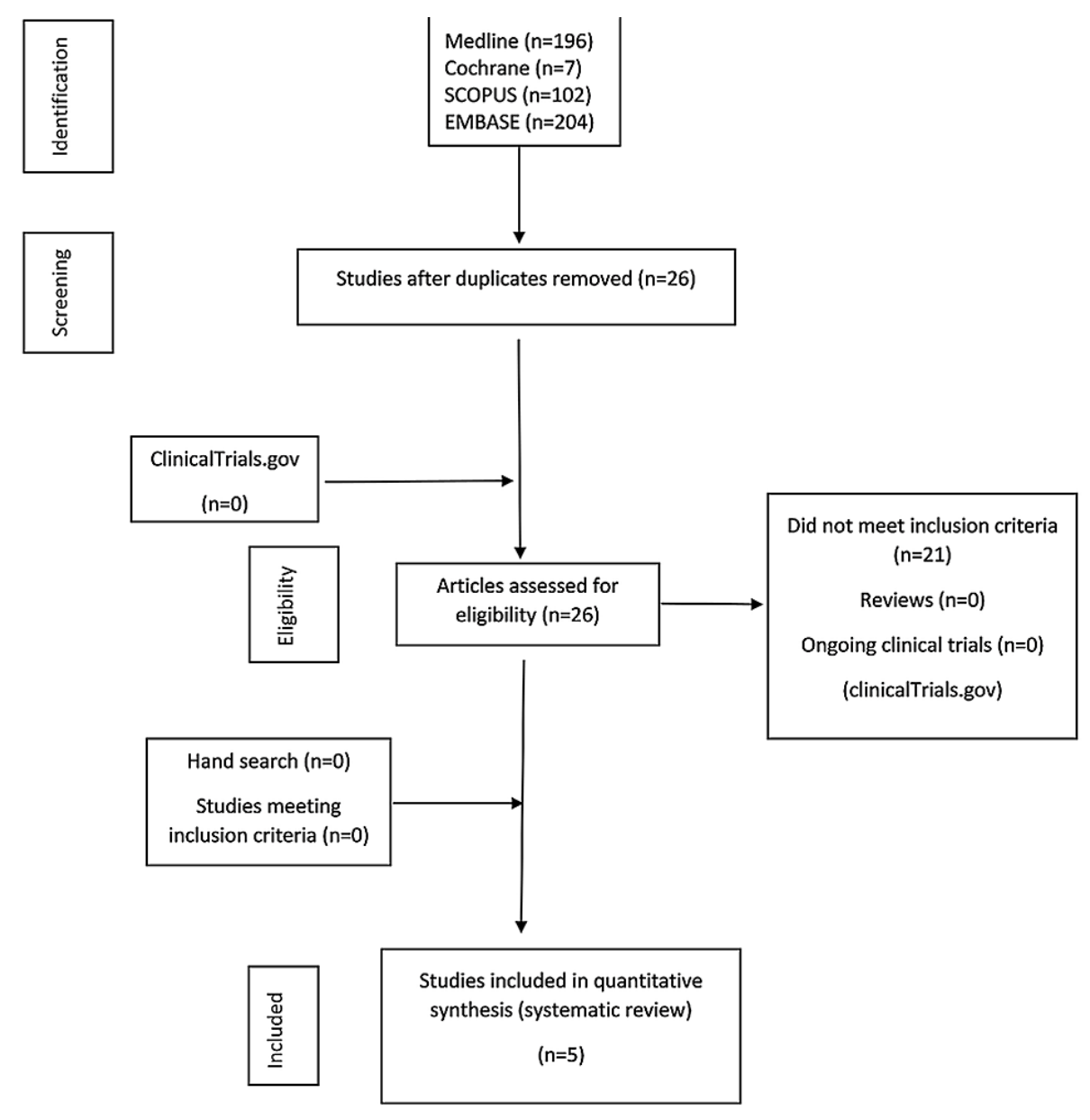

\section{FIGURE 1: Flowchart describing the systematic search and study selection process}

\section{Study characteristics}

Five relevant studies with 6575 patients were included. In studies that included both NVAF and VTE patients, data were extracted for VTE patients only [18-19]. In the study by Quan et al., the analysis was limited to major bleeding rates, as data on VTE recurrence were not reported [20]. For the number of VTE events in the study by Spyropoulos et al., we considered VTE events as diagnosed from imaging studies or VTE as primary inpatient diagnosis (Table 2) [21]. In the study by Sa et al., only patients with weight $\geqslant 20 \mathrm{~kg}$ were included [22]. Analysis of quality found scores of 7 of 9 for all reviewed studies, with each losing points for reporting of the adequacy of follow-up (Table 3).

\begin{tabular}{|c|c|c|c|c|c|c|}
\hline $\begin{array}{l}\text { Study and } \\
\text { study type }\end{array}$ & $\begin{array}{l}\text { Drugs } \\
\text { compared }\end{array}$ & $\begin{array}{l}\text { Total } \\
\text { patients }\end{array}$ & Weight & $\begin{array}{l}\text { Recurrent VTE } \\
\text { events }\end{array}$ & $\begin{array}{l}\text { Major bleeding } \\
\text { events }\end{array}$ & $\begin{array}{l}\text { Included for the study? If } \\
\text { no, why? }\end{array}$ \\
\hline $\begin{array}{l}\text { RECOVER } 1 \\
\text { and } 2 \\
\text { Randomized } \\
\text { double-blind } \\
\text { trial with } \\
\text { subgroup } \\
\text { analysis } \\
\text { based on } \\
\text { weight }\end{array}$ & $\begin{array}{l}\text { a. } \\
\text { Dabigatran } \\
\text { and b. } \\
\text { Warfarin for } \\
\text { VTE }\end{array}$ & $N=5107$ & $\begin{array}{l}\text { Subgroup weight } \\
>100 \mathrm{~kg}(\mathrm{~N}=832)\end{array}$ & $\begin{array}{l}\text { No interaction } \\
\text { between body } \\
\text { weight and primary } \\
\text { endpoints (recurrent } \\
\text { VTE and deaths) }\end{array}$ & NS & $\begin{array}{l}\text { No. Because there is no } \\
\text { separate data for morbidly } \\
\text { obese patients }\end{array}$ \\
\hline $\begin{array}{l}\text { AMPLIFY } \\
\text { randomized } \\
\text { double-blind } \\
\text { trial with } \\
\text { subgroup }\end{array}$ & $\begin{array}{l}\text { a. Apixaban } \\
\text { and b. } \\
\text { Warfarin for }\end{array}$ & $\mathrm{N}=5400$ & $\begin{array}{l}\text { Subgroup weight } \\
>100 \mathrm{~kg}\end{array}$ & $\begin{array}{l}\text { No interaction } \\
\text { between body } \\
\text { weight and primary }\end{array}$ & $\begin{array}{l}\text { No interaction } \\
\text { between body } \\
\text { weight and }\end{array}$ & $\begin{array}{l}\text { No. Because there is no } \\
\text { separate data for morbidly }\end{array}$ \\
\hline
\end{tabular}




\section{Cureus}

\begin{tabular}{|c|c|c|c|c|c|c|}
\hline $\begin{array}{l}\text { analysis } \\
\text { based on } \\
\text { weight }\end{array}$ & VTE & & $(\mathrm{N}=1017)$ & $\begin{array}{l}\text { endpoints (recurrent } \\
\text { VTE and deaths) }\end{array}$ & major bleeding & obese patients \\
\hline $\begin{array}{l}\text { EINSTEIN-PE } \\
\text { Randomized, } \\
\text { open-label, } \\
\text { with subgroup } \\
\text { analysis } \\
\text { based on } \\
\text { weight }\end{array}$ & $\begin{array}{l}\text { a. } \\
\text { Rivaroxaban } \\
\text { and b. } \\
\text { Warfarin for } \\
\text { PE }\end{array}$ & $\mathrm{N}=4833$ & $\begin{array}{l}\text { Subgroup } \\
\text { weight }>90 \mathrm{~kg} \\
(\mathrm{~N}=1355)\end{array}$ & $\begin{array}{l}\text { No interaction } \\
\text { between body } \\
\text { weight and primary } \\
\text { endpoint (recurrent } \\
\text { VTE) }\end{array}$ & $\begin{array}{l}\text { No interaction } \\
\text { between body } \\
\text { weight and } \\
\text { major bleeding }\end{array}$ & $\begin{array}{l}\text { No. Because there is no } \\
\text { separate data for morbidly } \\
\text { obese patients }\end{array}$ \\
\hline $\begin{array}{l}\text { EINSTEIN- } \\
\text { DVT } \\
\text { Randomized, } \\
\text { open-label, } \\
\text { with subgroup } \\
\text { analysis } \\
\text { based on } \\
\text { weight }\end{array}$ & $\begin{array}{l}\text { a. } \\
\text { Rivaroxaban } \\
\text { and b. } \\
\text { Warfarin for } \\
\text { VTE }\end{array}$ & $\mathrm{N}=3499$ & $\begin{array}{l}\text { Subgroup weight } \\
>90 \mathrm{~kg}(\mathrm{~N}=969)\end{array}$ & $\begin{array}{l}\text { No interaction } \\
\text { between body } \\
\text { weight and primary } \\
\text { endpoint (recurrent } \\
\text { VTE) }\end{array}$ & $\begin{array}{l}\text { No interaction } \\
\text { between body } \\
\text { weight and } \\
\text { major bleeding }\end{array}$ & $\begin{array}{l}\text { No. Because there is no } \\
\text { separate data for morbidly } \\
\text { obese patients }\end{array}$ \\
\hline $\begin{array}{l}\text { HOKUSAI VTE } \\
\text { Randomized, } \\
\text { double-blind } \\
\text { with subgroup } \\
\text { analysis } \\
\text { based on } \\
\text { weight }\end{array}$ & $\begin{array}{l}\text { a. Edoxaban } \\
\text { and b. } \\
\text { Warfarin for } \\
\text { VTE }\end{array}$ & $\mathrm{N}=4921$ & $\begin{array}{l}\text { Subgroup weight } \\
>100 \mathrm{~kg} \\
(\mathrm{~N}=1265)\end{array}$ & $\begin{array}{l}\text { No interaction } \\
\text { between body } \\
\text { weight and primary } \\
\text { endpoint (recurrent } \\
\text { VTE) }\end{array}$ & $\begin{array}{l}\text { No interaction } \\
\text { between body } \\
\text { weight and } \\
\text { major bleeding }\end{array}$ & $\begin{array}{l}\text { No. Because there is no } \\
\text { separate data for morbidly } \\
\text { obese patients }\end{array}$ \\
\hline $\begin{array}{l}\text { Kushnir et al. } \\
\text { Single-center, } \\
\text { retrospective } \\
\text { analysis }\end{array}$ & $\begin{array}{l}\text { a. DOAC: } \\
\text { Rivaroxaban } \\
\text { or Apixaban } \\
\text { and b. } \\
\text { Warfarin for } \\
\text { either VTE or } \\
\text { AF }\end{array}$ & $\begin{array}{l}\mathrm{N}=795 \\
\text { For VTE: } \\
\mathrm{R}: 152 \\
\mathrm{~A}: 47 \\
\mathrm{~W}: 167\end{array}$ & $\mathrm{BMl}>40 \mathrm{~kg} / \mathrm{m}^{2}$ & $\begin{array}{l}\text { For VTE: Similar } \\
\text { incidence of } \\
\text { recurrent VTE } \\
\text { between Apixaban } \\
(2.1 \%) \text {, rivaroxaban } \\
(2 \%) \text {, and warfarin } \\
(1.2 \%) \text { cohort }\end{array}$ & $\begin{array}{l}\text { For VTE: Similar } \\
\text { incidence of } \\
\text { major bleeding } \\
\text { events in } 2 \\
\text { cohorts }\end{array}$ & Yes \\
\hline $\begin{array}{l}\text { Syropoulos et } \\
\text { al. } \\
\text { Retrospective } \\
\text { study of } 2 \\
\text { databases }\end{array}$ & $\begin{array}{l}\text { a. } \\
\text { Rivaroxaban } \\
\text { and b. } \\
\text { Warfarin for } \\
\text { VTE }\end{array}$ & $\begin{array}{l}N=5780 \\
R: 2890 \\
W: 2890\end{array}$ & $\mathrm{BMl}>40 \mathrm{~kg} / \mathrm{m}^{2}$ & $\begin{array}{l}\text { Similar incidence of } \\
\text { recurrent VTE } \\
\text { between } \\
\text { Rivaroxaban (16.8\%) } \\
\text { and warfarin (15.9\%) }\end{array}$ & $\begin{array}{l}\text { Similar } \\
\text { incidence of } \\
\text { major bleeding }\end{array}$ & $\begin{array}{l}\text { Yes. But only patients that } \\
\text { fulfilled the definition of } \\
\text { recurrent VTE as VTE events } \\
\text { as diagnosed from imaging } \\
\text { studies or VTE as primary } \\
\text { inpatient diagnosis were } \\
\text { taken. }\end{array}$ \\
\hline $\begin{array}{l}\text { Coons et al. } \\
\text { Retrospective } \\
\text { study }\end{array}$ & $\begin{array}{l}\text { a. DOAC: } \\
\text { Apixaban or } \\
\text { Rivaroxaban } \\
\text { or } \\
\text { Dabigatran } \\
\text { and b. } \\
\text { Warfarin for } \\
\text { VTE }\end{array}$ & $\begin{array}{l}N=1840 \\
\text { DOAC: } \\
632 \\
W: 1208\end{array}$ & $\begin{array}{l}300 \mathrm{~kg}>\text { Weight } \\
>100 \mathrm{~kg}\end{array}$ & $\begin{array}{l}\text { No significant } \\
\text { difference between } \\
\text { DOAC }(6.5 \%) \text { and } \\
\text { warfarin }(6.4 \%) \text { in } \\
\text { the prevention of } \\
\text { recurrence of VTE }\end{array}$ & $\begin{array}{l}\text { No significant } \\
\text { difference in } \\
\text { major bleeding } \\
\text { events }\end{array}$ & $\begin{array}{l}\text { No. Because there is no } \\
\text { separate data for morbidly } \\
\text { obese patients }\end{array}$ \\
\hline $\begin{array}{l}\text { Perales et al. } \\
\text { Retrospective } \\
\text { review at } 2 \\
\text { academic } \\
\text { medical } \\
\text { centers }\end{array}$ & $\begin{array}{l}\text { a. } \\
\text { Rivaroxaban } \\
\text { and b. } \\
\text { Warfarin for } \\
\text { VTE and AF }\end{array}$ & $\begin{array}{l}\mathrm{N}=176 \mathrm{ln} \\
\text { VTE } \\
\text { patients: } \\
\text { R: } 47 \mathrm{~W}: \\
62\end{array}$ & $\begin{array}{l}\mathrm{BMl}>40 \mathrm{~kg} / \mathrm{m}^{2} \\
\text { Weight }>120 \mathrm{~kg}\end{array}$ & $\begin{array}{l}\text { In VTE patients: No } \\
\text { significant } \\
\text { difference in the } \\
\text { recurrence of VTE in } \\
\text { rivaroxaban (4\%) } \\
\text { and warfarin }(6 \%)\end{array}$ & $\begin{array}{l}\text { In VTE patients } \\
\text { No significant } \\
\text { difference in } \\
\text { bleeding events } \\
\text { in rivaroxaban } \\
(6 \%) \text { and } \\
\text { warfarin }(3 \%)\end{array}$ & Yes \\
\hline $\begin{array}{l}\text { Kalani et al. } \\
\text { Retrospective } \\
\text { study }\end{array}$ & $\begin{array}{l}\text { a. DOACs: } \\
\text { Apixaban or } \\
\text { Rivaroxaban } \\
\text { or } \\
\text { Dabigatran } \\
\text { b. Warfarin }\end{array}$ & $\begin{array}{l}\mathrm{N}=180 \text { No } \\
\text { separate } \\
\text { data for } \\
\text { VTE and } \\
\text { AF } \\
\text { patients }\end{array}$ & $\begin{array}{l}\mathrm{BMl}>40 \mathrm{~kg} / \mathrm{m}^{2} \\
\text { Weight }>120 \mathrm{~kg}\end{array}$ & $\begin{array}{l}\text { For combined VTE } \\
\text { and AF: No } \\
\text { significant } \\
\text { difference in the } \\
\text { primary outcome } \\
\text { (composite of }\end{array}$ & $\begin{array}{l}\text { Similar } \\
\text { bleeding events } \\
\text { in DOAC and } \\
\text { warfarin group }\end{array}$ & $\begin{array}{l}\text { No. Because there is no } \\
\text { separate data for VTE and } \\
\text { AF patients }\end{array}$ \\
\hline
\end{tabular}




\section{Cureus}

\begin{tabular}{|c|c|c|c|c|c|c|}
\hline & $\begin{array}{l}\text { for VTE and } \\
\text { AF }\end{array}$ & $\begin{array}{l}\text { DOAC: } 90 \\
\text { W:90 }\end{array}$ & & $\begin{array}{l}\text { stroke, TIA, VTE, PE) } \\
\text { in both arms. }\end{array}$ & & \\
\hline $\begin{array}{l}\text { Quann et al. } \\
\text { Retrospective } \\
\text { sub-study }\end{array}$ & $\begin{array}{l}\text { a. DOAC: } \\
\text { Apixaban or } \\
\text { Rivaroxaban } \\
\text { or } \\
\text { Dabigatran } \\
\text { and b. TT: } \\
\text { LMWH or } \\
\text { warfarin }\end{array}$ & $\begin{array}{l}\mathrm{N}=187 \\
\text { DOAC: } \\
109 \text { TT: } \\
78\end{array}$ & Weight $>120 \mathrm{~kg}$ & $\begin{array}{l}\text { Overall rate of } \\
\text { recurrent VTE out to } \\
1 \text { year was } 0.006 \\
\text { events/ patient-year }\end{array}$ & $\begin{array}{l}\text { Similar } \\
\text { bleeding events } \\
\text { in DOAC (8.3\%) } \\
\text { and } T(11.5 \%)\end{array}$ & $\begin{array}{l}\text { Yes. But only bleeding } \\
\text { event taken because the } \\
\text { primary outcome was the } \\
\text { overall rate of recurrent } \\
\text { VTE. }\end{array}$ \\
\hline $\begin{array}{l}\text { Tittl et al. } \\
\text { Prospective } \\
\text { Dresden } \\
\text { registry study }\end{array}$ & $\begin{array}{l}\text { DOAC in } \\
\text { different BMI } \\
\text { groups for } \\
\text { VTE and AF }\end{array}$ & $\mathrm{N}=3432$ & $\begin{array}{l}6 \text { groups } \\
\text { stratified } \\
\text { according to } \\
\text { BMI (in } \mathrm{kg} / \mathrm{m}^{2} \text { ) a. } \\
<18.5 \text { b. } 18.5- \\
24.9 \text { c. } 25-29.9 \text { d. } \\
30-34.9 \text { e. } 35- \\
39.9 \text { f. }>40\end{array}$ & $\begin{array}{l}\text { Thromboembolic } \\
\text { rate: In BMI> 40: } \\
0.49 \text { events/100 } \\
\text { patient-years }\end{array}$ & $\begin{array}{l}\text { Similar major } \\
\text { bleeding rates } \\
\text { in between } \\
\text { those with } \\
\mathrm{BMl}>30 \text { and } \\
<30 \mathrm{~kg} / \mathrm{m}^{2}\end{array}$ & $\begin{array}{l}\text { No. Because it compared } \\
\text { the clinical outcome of } \\
\text { DOAC in different BMI } \\
\text { groups }\end{array}$ \\
\hline $\begin{array}{l}\text { Aloi et al. } \\
\text { Retrospective } \\
\text { analysis }\end{array}$ & $\begin{array}{l}\text { DOAC for } \\
\text { VTE in } \\
\text { patients with } \\
\text { weight } \geq \\
120 \mathrm{~kg} \\
\text { compared to } \\
\text { that with } \\
<120 \mathrm{~kg}\end{array}$ & $\mathrm{~N}=1196$ & $\begin{array}{l}\text { Two groups } \\
\text { according to } \\
\text { weight a. } \geq \\
120 \mathrm{~kg} \text { b. }<120 \\
\mathrm{~kg}\end{array}$ & $\begin{array}{l}\text { No significant } \\
\text { difference in the } \\
\text { recurrence of VTE in } \\
\text { both groups }\end{array}$ & NS & $\begin{array}{l}\text { No. Because it compared } \\
\text { clinical outcome of only } \\
\text { DOAC in } 2 \text { different weight } \\
\text { group }\end{array}$ \\
\hline $\begin{array}{l}\text { Netly et al. } \\
\text { Retrospective } \\
\text { study }\end{array}$ & $\begin{array}{l}\text { DOAC in } \\
\text { different BMI } \\
\text { groups for } \\
\text { AF, VTE, and } \\
\text { VTE chronic } \\
\text { prophylaxis }\end{array}$ & $N=113$ & $\begin{array}{l}3 \text { groups } \\
\text { stratified } \\
\text { according to } \\
\left.\text { BMI (in } \mathrm{kg} / \mathrm{m}^{2}\right) \text { : } \\
\text { a. }<30 \text { b. } 30-40> \\
40\end{array}$ & $\begin{array}{l}\text { No statistically } \\
\text { significant } \\
\text { differences in } 3 \\
\text { groups. BMl }>40 \mathrm{~kg} \\
/ \mathrm{m}^{2} \text { had highest } \\
\text { thrombotic events. }\end{array}$ & $\begin{array}{l}\text { No statistically } \\
\text { significant } \\
\text { differences in } 3 \\
\text { groups. BMl> } \\
40 \mathrm{~kg} / \mathrm{m}^{2} \text { had } \\
\text { lowest bleeding } \\
\text { events. }\end{array}$ & $\begin{array}{l}\text { No. Because it compared } \\
\text { the clinical outcome of only } \\
\text { DOAC in different BMI } \\
\text { groups }\end{array}$ \\
\hline $\begin{array}{l}\text { Sa et al. } \\
\text { Retrospective } \\
\text { study }\end{array}$ & $\begin{array}{l}\text { a. DOAC: } \\
\text { Rivaroxaban, } \\
\text { Apixaban } \\
\text { and } \\
\text { edoxaban b. } \\
\text { Warfarin }\end{array}$ & $\begin{array}{l}\text { N=133 } \\
\text { DOAC: } 71 \\
\text { W: } 62\end{array}$ & $\begin{array}{l}\text { a. } 120>\mathrm{Weight} \geq \\
120 \mathrm{~kg} \mathrm{~b} .30 \mathrm{~kg} / \\
\mathrm{m}^{2}>\mathrm{BMI} \geq 30 \\
\mathrm{~kg} / \mathrm{m}^{2} \text { only } \\
\text { weight } \geq 120 \mathrm{~kg} \\
\text { selected }\end{array}$ & $\begin{array}{l}\text { No significant } \\
\text { difference in the } \\
\text { recurrence of VTE in } \\
\text { both groups }\end{array}$ & $\begin{array}{l}\text { Similar major } \\
\text { bleeding rates } \\
\text { between two } \\
\text { groups }\end{array}$ & $\begin{array}{l}\text { Yes. But only data from the } \\
\text { weight group } \geq 120 \mathrm{~kg} \text {. }\end{array}$ \\
\hline
\end{tabular}

\section{TABLE 2: Prior studies comparing DOACs in obesity}

VTE, venous thromboembolism, AF, atrial fibrillation, BMI, body mass index; PE, pulmonary embolism; A, apixaban; NS, not specified; R, rivaroxaban; $\mathrm{W}$, warfarin; TIA: transient ischemic attack; DOAC: directly acting anticoagulants (including apixaban, rivaroxaban, and dabigatran), $\mathrm{TT}$ : traditional therapy (including warfarin and low molecular weight heparin (LMWH)) 


\section{Cureus}

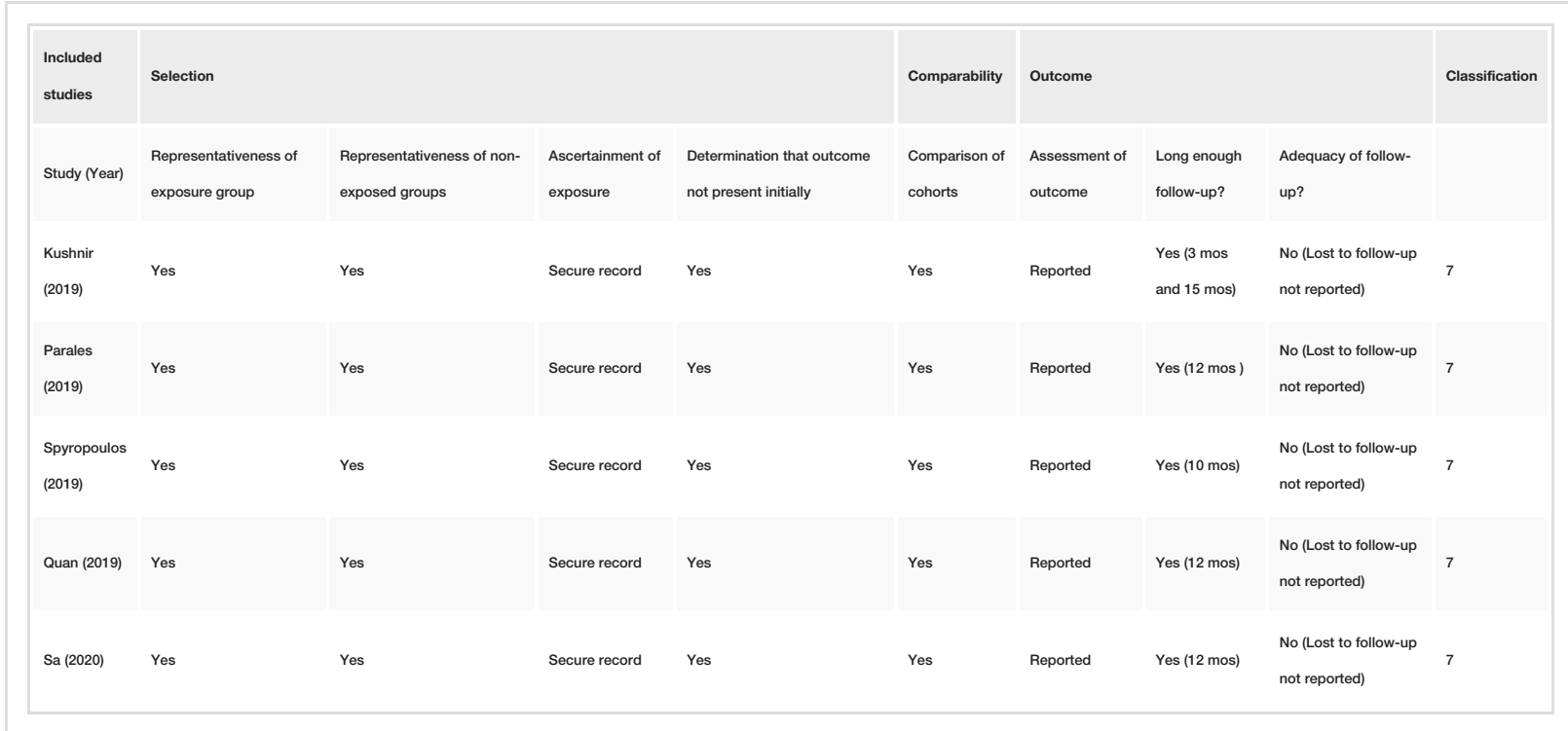

TABLE 3: Modified Newcastle-Ottawa Quality Assessment Scale for retrospective studies

\section{Recurrent VTE}

Recurrent VTE occurred in 95 of 3207 (2.96\%) patients in the DOAC group and 81 of 3181 (2.54\%) patients in the VKA and LMWH group (OR: 1.17; $95 \%$ CI 0.87 to 1.59 , p=.30) (Figure 2). Slightly higher rates of VTE were mainly driven by the Spyropoulos et al. study [21], possibly driven by the fact that $7 \%$ of their population had malignancy and another $7 \%$ had chronic kidney disease. Sensitivity analysis performed excluding this study found VTE recurrence in DOACs of 2.52\% and VKA/LMWH of 2.06\% (OR: 1.29; 95\% CI: 0.41 to 3.99, $\mathrm{p}=.66$ ) (Figure 3).

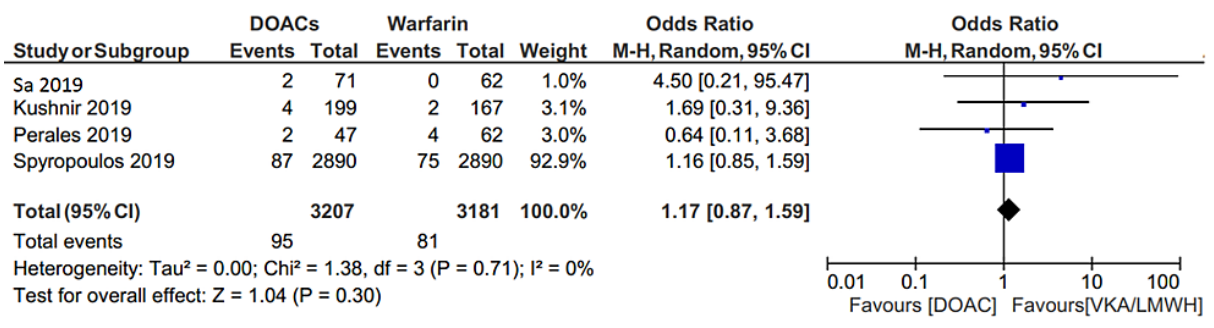

FIGURE 2: Forest plot showing the comparison of recurrent VTE between two groups

VTE, venous thromboembolism

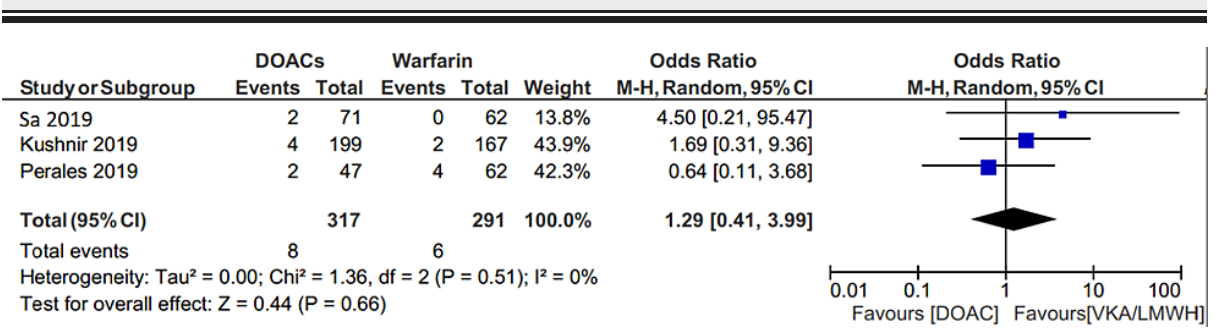

FIGURE 3: Forest plot showing a comparison of recurrent VTE between two groups excluding the study by Spyropoulos et al.

VTE, venous thromboembolism 


\section{Major bleeding}

Major bleeding occurred in 63 of 3316 (1.89\%) patients in the DOAC group, and 83 of 3259(2.54\%) patients in the VKA or LMWH group (OR: 0.74; 95\% CI: 0.53 to 1.03, p=.08) (Figure 4).

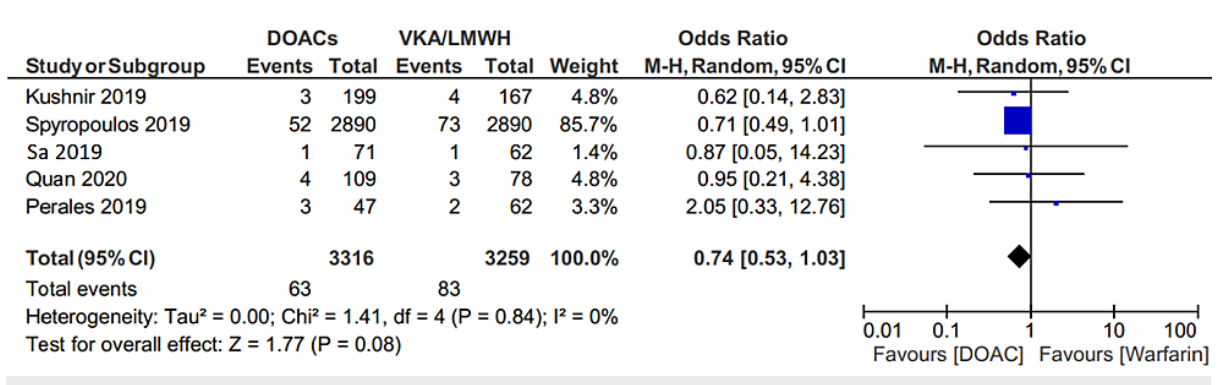

\section{FIGURE 4: Forest plot showing a comparison of major bleeding between} two groups

Although the majority of the included studies compared factor Xa inhibitor (apixaban and rivaroxaban) with warfarin, the study by Quan et al. [20] also included dabigatran with other DOACs. Given that the Quan et al. study also included dabigatran, we performed a sensitivity analysis excluding this study to isolate the Xa inhibitors and found similar results.

Additional sensitivity analysis comparing the factor Xa inhibitors apixaban, rivaroxaban, and edoxaban to warfarin showed similar rates of major bleeding (59/3207 (1.83\%) patients in the factor Xa inhibitor group versus $80 / 3181$ (2.51\%) patients in the VKA group (OR 0.73; 95\% CI 0.52 to $1.03, \mathrm{p}=.07$ ) (Figure 5).

\begin{tabular}{|c|c|c|c|c|c|c|c|c|}
\hline Study or Subgroup & \multicolumn{2}{|c|}{ DOACs } & \multicolumn{2}{|c|}{ VKA/LMWH } & Weight & $\begin{array}{c}\text { Odds Ratio } \\
\text { M-H, Random, } 95 \% \mathrm{Cl}\end{array}$ & \multicolumn{2}{|c|}{$\begin{array}{c}\text { Odds Ratio } \\
\text { M-H, Random, } 95 \% \mathrm{Cl}\end{array}$} \\
\hline Kushnir 2019 & 3 & 199 & 4 & 167 & $5.1 \%$ & $0.62[0.14,2.83]$ & & \\
\hline Spyropoulos 2019 & 52 & 2890 & 73 & 2890 & $90.0 \%$ & $0.71[0.49,1.01]$ & & \\
\hline Sa 2019 & 1 & 71 & 1 & 62 & $1.5 \%$ & $0.87[0.05,14.23]$ & & \\
\hline Perales 2019 & 3 & 47 & 2 & 62 & $3.5 \%$ & $2.05[0.33,12.76]$ & & \\
\hline Total $(95 \% \mathrm{Cl})$ & & 3207 & & 3181 & $100.0 \%$ & $0.73[0.52,1.03]$ & & \\
\hline Total events & 59 & & 80 & & & & & \\
\hline $\begin{array}{l}\text { Heterogeneity: } \text { Tau }^{2} \\
\text { Test for overall effec }\end{array}$ & $\begin{array}{l}.00 ; \mathrm{Chi}^{2} \\
=1.80(\end{array}$ & $\begin{array}{l}=1.30, \\
P=0.07\end{array}$ & $\begin{array}{l}\mathrm{df}=3(\mathrm{P} \\
7)\end{array}$ & $=0.73)$ & $;\left.\right|^{2}=0 \%$ & & \begin{tabular}{|ll}
0.01 & 0.1 \\
Favours [DOAC]
\end{tabular} & $\begin{array}{|cc|}10 & 10 \\
\text { Favours [Warfarin] }\end{array}$ \\
\hline
\end{tabular}

FIGURE 5: Forest plot showing a comparison of major bleeding between two groups excluding the study by Quan et al.

\section{Discussion}

In this study, we found a similar incidence of recurrent VTE and major bleeding with both DOACs and VKA/LMWH in morbidly obese patients. Our study is one of the few studies to date examining the safety and efficacy profiles of DOAC in these patients.

Our results are consistent with a prior meta-analysis looking at VTE prophylaxis in obese patients in a postarthroplasty setting that compared DOACs to LMWH. Our data are also consistent with a prior meta-analysis of DOACs for the treatment of VTE in non-morbidly obese patients [23]. Although our findings are similar to another meta-analysis by Elshafei et al., our study has clear data presentation and summarization of the study and sensitivity analysis [24]. Our results also align with data from a study comparing DOACs with VKA in morbidly obese patients (BMI $>40 \mathrm{~kg} / \mathrm{m}^{2}$ and weight $>120 \mathrm{~kg}$ ) with NVAF that showed similar efficacy in the prevention of VTE recurrence, systemic embolism, and a similar bleeding risk [14,18].

We notice that the rate of recurrent VTE in our study was higher in both arms $(2.9 \%$, for DOACs, $2.5 \%$ in VKAs) than reported in the prior DOACs study in non-obese patients, which presumably were randomized trials. We hypothesize that this may be related to an increased risk of recurrent VTE in obese individuals [25]. There could be a higher incidence in the studies included here because the inclusion criteria were less stringent than in randomized trials and thus older and/or sicker patients were in the cohort studies. This study underscores the need for further modifications of therapy to reduce the high VTE recurrence rate irrespective of whether the patient is on a DOAC or VKA. In the case of DOACs, studying dosing continuously at $15 \mathrm{mg}$ bid of rivaroxaban or $10 \mathrm{mg}$ bid of apixaban beyond the respective "lead-in" periods in morbidly obese patients would seem worthy of a clinical trial but given the relatively low recurrence rate, this would have to be a very large multi-institutional randomized clinical trial. Given that our study includes only 
patients with BMI $\geqslant 40 \mathrm{~kg} / \mathrm{m}^{2}$, our data helps understand the effect of extremes of body weight on the efficacy of DOACs. Further studies are needed to determine if there is a "ceiling effect" precluding efficacy, possibly at a BMI of $>60 \mathrm{~kg} / \mathrm{m}^{2}$, as these patients were under-represented in our study.

In terms of safety data, we observed a similar lower rate of major bleeding with DOACs as compared with VKA or LMWH in morbidly obese patients. Previous studies examining the pharmacokinetic attributes of rivaroxaban have found a similar peak concentration, volume of distribution, and half-life between patients who weighed more than $120 \mathrm{~kg}$ and those who weighed between 70 and $80 \mathrm{~kg}$ [26]. However, in patients taking apixaban, higher body weight ( $>120 \mathrm{~kg}$ and $\mathrm{BMI} \geqslant 30 \mathrm{~kg} / \mathrm{m}^{2}$ ) was associated with a lower mean peak concentration, higher volume of distribution, lower drug exposure, and shorter mean half-life compared with normal weight $\left(65-85 \mathrm{~kg}\right.$ and $\left.\mathrm{BMI} \leqslant 30 \mathrm{~kg} / \mathrm{m}^{2}\right)$ [27]. These differences in pharmacokinetic properties by body weights do not seem to be consistent across various DOACs and, therefore, the clinical implications also remain unclear. Finally, although lower bleeding rates have been reported in prior studies with DOACs in major studies specifically with apixaban [23], we were unable to examine bleeding rates separately for each DOACs.

Given that some clinicians are already using DOACs, even in obese and morbidly obese patients, after a careful discussion of risk-benefit profiles with patients [4], our data give additional credence to this practice. Although only randomized controlled trials can make a definitive conclusion on the safety and efficacy of DOACs versus VKA/LMWH, these trials are unlikely to be conducted. Therefore, our data represent the best available evidence to guide clinical practice at this point.

Other strengths of our study include a large sample size with low heterogeneity $\left(\mathrm{I}^{2}=0 \%\right)$ and a relatively uniform reporting of outcomes.

\section{Limitations}

The limitations of our study include the inclusion of non-randomized observationals that often have much large effect sizes than randomized trials, likely because of selection biases. In the warfarin arm, the time to therapeutic range was unknown due to missing long-term INR data. Similarly, included studies did not report measurement of DOAC levels to ensure therapeutic plasma drug levels. This could have potentially led to the under-dosing of DOACs. For example, a prior study found the prevalence of under-dosing with apixaban at $24.5 \%$ compared with $12.8 \%$ with rivaroxaban and $14 \%$ with dabigatran. Five percent of patients that were under-dosed with apixaban was related to patient body weight, unlike rivaroxaban and dabigatran where weight had no effect on under-dosing [28]. As we have noted, obesity may have a different impact on the pharmacokinetics of various DOACs. The approach of lumping patients treated with different DOACs as one group is unjustified, as it might obscure potential differences among the DOACs. Each DOAC should be treated as a separate group. Also, it is unclear what percentage of patients had adherence to the treatment in both arms. It is also unclear as to what percentage of included patients underwent bariatric surgery given data suggesting variable plasma drug levels of DOACs in bariatric surgery patients [29]. Finally, we were unable to ascertain the impact of comorbidities on both safety and efficacy outcomes. We were not able to determine what percentage of patients may have been on OCPs, had prior VTE events, or had cancer that was not captured by retrospective review.

\section{Conclusions}

In this systematic review and meta-analysis of retrospective studies evaluating DOACs versus VKA/LMWH in morbidly obese patients, we demonstrate that both the safety and efficacy of DOACs might be similar to VKA/LMWH.

\section{Additional Information \\ Disclosures}

Human subjects: All authors have confirmed that this study did not involve human participants or tissue. Animal subjects: All authors have confirmed that this study did not involve animal subjects or tissue. Conflicts of interest: In compliance with the ICMJE uniform disclosure form, all authors declare the following: Payment/services info: All authors have declared that no financial support was received from any organization for the submitted work. Financial relationships: All authors have declared that they have no financial relationships at present or within the previous three years with any organizations that might have an interest in the submitted work. Other relationships: All authors have declared that there are no other relationships or activities that could appear to have influenced the submitted work.

\section{Acknowledgements}

We would like to thank Dr. Anthony Donato MD for his valuable suggestions and expert opinion in the subsequent editing of this manuscript.

\section{References}


1. Kearon C, Akl EA, Ornelas J, et al.: Antithrombotic therapy for VTE disease: CHEST guideline and expert panel report. Chest. 2016, 149:315-52. 10.1016/j.chest.2015.11.026

2. Barnes GD, Lucas E, Alexander GC, Goldberger ZD: National trends in ambulatory oral anticoagulant use . Am J Med. 2015, 128:1300-5.e2. 10.1016/j.amjmed.2015.05.044

3. Bauersachs R, Berkowitz SD, Brenner B, et al.: Oral rivaroxaban for symptomatic venous thromboembolism. N Engl J Med. 2010, 363:2499-510. 10.1056/NEJMoa1007903

4. Wang TF, Carrier M: How I treat obese patients with oral anticoagulants . Blood. 2020, 19:904-911. 10.1182/blood.2019003528

5. Eichinger S, Hron G, Bialonczyk C, et al.: Overweight, obesity, and the risk of recurrent venous thromboembolism. Arch Intern Med. 2008, 168:1678-83. 10.1001/archinte.168.15.1678

6. Pathak R, Karmacharya P, Giri S, et al.: Meta-analysis on efficacy and safety of new oral anticoagulants for venous thromboembolism prophylaxis in overweight and obese postarthroplasty patients. Blood Coagul Fibrinolysis. 2015, 26:635-42. 10.1097/MBC.0000000000000327

7. Martin K, Beyer-Westendorf J, Davidson BL, Huisman MV, Sandset PM, Moll S: Use of the direct oral anticoagulants in obese patients: guidance from the SSC of the ISTH. J Thromb Haemost. 2016, 14:1308-13. 10.1111/jth.13323

8. Di Minno MN, Lupoli R, Di Minno A, Ambrosino P, Scalera A, Dentali F: Effect of body weight on efficacy and safety of direct oral anticoagulants in the treatment of patients with acute venous thromboembolism: a meta-analysis of randomized controlled trials. Ann Med. 2015, 47:61-8. 10.3109/07853890.2014.982064

9. Coons JC, Albert L, Bejjani A, Iasella CJ: Effectiveness and safety of direct oral anticoagulants versus warfarin in obese patients with acute venous thromboembolism. Pharmacotherapy. 2020, 40:204-10. 10.1002/phar.2369

10. Nutescu EA, Spinler SA, Wittkowsky A, Dager WE: Low-molecular-weight heparins in renal impairment and obesity: available evidence and clinical practice recommendations across medical and surgical settings. Ann Pharmacother. 2009, 43:1064-83. 10.1345/aph.1L194

11. Beller EM, Glasziou PP, Altman DG, et al.: PRISMA for abstracts: reporting systematic reviews in journal and conference abstracts. PLoS Med. 2013, 10:e1001419. 10.1371/journal.pmed.1001419

12. Aloi KG, Fierro JJ, Stein BJ, Lynch SM, Shapiro RJ: Investigation of direct-acting oral anticoagulants and the incidence of venous thromboembolism in patients weighing $\geqslant 120 \mathrm{~kg}$. J Pharm Pract. 2021, 34:64-9. 10.1177/0897190019854578

13. Kalani C, Awudi E, Alexander T, Udeani G, Surani S: Evaluation of the efficacy of direct oral anticoagulants (DOACs) in comparison to warfarin in morbidly obese patients. Hosp Pract (1995). 2019, 47:181-5. 10.1080/21548331.2019.1674586

14. Tittl L, Endig S, Marten S, Reitter A, Beyer-Westendorf I, Beyer-Westendorf J: Impact of BMI on clinical outcomes of NOAC therapy in daily care - results of the prospective Dresden NOAC Registry (NCT01588119). Int J Cardiol. 2018, 262:85-91. 10.1016/j.ijcard.2018.03.060

15. Netley J, Howard K, Wilson W: Effects of body mass index on the safety and effectiveness of direct oral anticoagulants: a retrospective review. J Thromb Thrombolysis. 2019, 48:359-65. 10.1007/s11239-01901857-2

16. Schulman S, Kearon C: Definition of major bleeding in clinical investigations of antihemostatic medicinal products in non-surgical patients. J Thromb Haemost. 2005, 3:692-4. 10.1111/j.1538-7836.2005.01204.x

17. Ottawa Hospital Research Institute. The Newcastle-Ottawa Scale (NOS) for assessing the quality of nonrandomised studies in meta-analyses. (2020). Accessed: April 21, 2020: http://www.ohri.ca/programs/clinical_epidemiology/oxford.asp.

18. Perales IJ, San Agustin K, DeAngelo J, Campbell AM: Rivaroxaban versus warfarin for stroke prevention and venous thromboembolism treatment in extreme obesity and high body weight. Ann Pharmacother. 2020, 54:344-50. 10.1177/1060028019886092

19. Kushnir M, Choi Y, Eisenberg R, et al.: Efficacy and safety of direct oral factor Xa inhibitors compared with warfarin in patients with morbid obesity: a single-centre, retrospective analysis of chart data. Lancet Haematol. 2019, 6:359-365. 10.1016/S2352-3026(19)30086-9

20. Quan S, Smith J, Wu C, Koshman SL, Nguyen B, Bungard TJ: Anticoagulant therapies and outcomes in obese patients with acute venous thromboembolism. Thromb Res. 2020, 187:56-62. 10.1016/j.thromres.2020.01.011

21. Spyropoulos AC, Ashton V, Chen YW, Wu B, Peterson ED: Rivaroxaban versus warfarin treatment among morbidly obese patients with venous thromboembolism: comparative effectiveness, safety, and costs. Thromb Res. 2019, 182:159-66. 10.1016/j.thromres.2019.08.021

22. Almeida Sa R, Al-Ani F, Lazo-Langner A, Louzada ML: Efficacy and safety of direct oral anticoagulants in obese patients with venous thromboembolism. Blood. 2019, 134:3675. 10.1182/blood-2019-121765

23. Aryal MR, Gosain R, Donato A, et al.: Systematic review and meta-analysis of the efficacy and safety of apixaban compared to rivaroxaban in acute VTE in the real world. Blood Adv. 2019, 3:2381-7. 10.1182/bloodadvances.2019000572

24. Elshafei MN, Mohamed MFH, El-Bardissy A, Ahmed MB, Abdallah I, Elewa H, Danjuma M: Comparative effectiveness and safety of direct oral anticoagulants compared to warfarin in morbidly obese patients with acute venous thromboembolism: systematic review and a meta-analysis. J Thromb Thrombolysis. 2021, 51:388-96. 10.1007/s11239-020-02179-4

25. Cushman M: Epidemiology and risk factors for venous thrombosis . Semin Hematol. 2007, 44:62-9. 10.1053/j.seminhematol.2007.02.004

26. Kubitza D, Becka M, Zuehlsdorf M, Mueck W: Body weight has limited influence on the safety, tolerability, pharmacokinetics, or pharmacodynamics of rivaroxaban (BAY 59-7939) in healthy subjects. J Clin Pharmacol. 2007, 47:218-26. 10.1177/0091270006296058

27. Upreti VV, Wang J, Barrett YC, et al.: Effect of extremes of body weight on the pharmacokinetics, pharmacodynamics, safety and tolerability of apixaban in healthy subjects. Br J Clin Pharmacol. 2013, 76:908-16. 10.1111/bcp.12114

28. Moudallel S, Steurbaut S, Cornu P, Dupont A: Appropriateness of DOAC prescribing before and during 


\section{Cureus}

hospital admission and analysis of determinants for inappropriate prescribing. Front Pharmacol. 2018,

9:1220. 10.3389/fphar.2018.01220

29. Moll S, Martin KA: Anticoagulant drug choice in patients who have had bariatric surgery - presently, DOACs are not the preferred choice. Thromb Res. 2018, 163:196-9. 10.1016/j.thromres.2018.01.027 